\title{
Exact solution of a percolation analog for the many-body localization transition
}

\author{
Sthitadhi Roy, ${ }^{1,2, *}$ David E. Logan, ${ }^{1, \dagger}$ and J. T. Chalker ${ }^{2, *}$ \\ ${ }^{1}$ Physical and Theoretical Chemistry, Oxford University, South Parks Road, Oxford OX1 3QZ, United Kingdom \\ ${ }^{2}$ Rudolf Peierls Centre for Theoretical Physics, Clarendon Laboratory, Oxford University, Parks Road, Oxford OX1 3PU, United Kingdom
}

(Received 8 January 2019; published 27 June 2019)

\begin{abstract}
We construct and solve a classical percolation model with a phase transition that we argue acts as a proxy for the quantum many-body localization transition. The classical model is defined on a graph in the Fock space of a disordered, interacting quantum spin chain, using a convenient choice of basis. Edges of the graph represent matrix elements of the spin Hamiltonian between pairs of basis states that are expected to hybridize strongly. At weak disorder, all nodes are connected, forming a single cluster. Many separate clusters appear above a critical disorder strength, each typically having a size that is exponentially large in the number of spins but a vanishing fraction of the Fock-space dimension. We formulate a transfer matrix approach that yields an exact value $v=2$ for the localization length exponent, and also use complete enumeration of clusters to study the transition numerically in finite-sized systems.
\end{abstract}

DOI: 10.1103/PhysRevB.99.220201

Insights into quantum many-body systems can be gained at a variety of levels from studying classical problems. An exact equivalence is provided by the well-known mapping between ground-state properties of a quantum system and finitetemperature behavior in a classical system in one higher dimension [1,2]. Qualitative understanding in quantum systems may, however, be derived from classical models in other ways, phenomenological in nature and rooted in physical argument. For example, the analogy between Anderson localization and classical percolation provides a picture which is particularly useful in the context of the integer quantum Hall effect [3-5]. Many-body localization transitions [6-14] are currently of great interest. They occur in highly excited states and concern dynamical properties of quantum systems. They are therefore not generally expected to admit exact mappings onto classical problems. The question of whether or how classical statistical mechanical models can be constructed that mimic aspects of such phase transitions is thus naturally of fundamental interest.

In this Rapid Communication we formulate a classical percolation problem inspired by the quantum mechanics of a disordered spin system. The percolation problem is defined on a graph in the Fock space of the spin chain. We find that there exists a percolation transition [15] in the classical model which mimics certain aspects of the many-body localization transition in the quantum model. In particular, our choice of diagnostic for the percolation transition is motivated by the behavior across the many-body localization transition of the Fock-space participation entropies of eigenstates of the quantum system [11,16].

Remarkably, the classical percolation problem arising in this way from a commonly studied spin model with local

\footnotetext{
*sthitadhi.roy@chem.ox.ac.uk

†david.logan@chem.ox.ac.uk

¥john.chalker@physics.ox.ac.uk
}

interactions, admits an exact solution using a transfer matrix. This allows us to extract analytically the critical disorder strength and the value $v=2$ for the localization length exponent. In addition, we corroborate and extend our results by enumerating Fock-space clusters exactly for finite-sized systems. Our approach is complementary to works that use a phenomenological renormalization group [17-21] or the semiclassical limit of Clifford circuits [22] to construct a classical percolation problem in real space, in that we work entirely in Fock space (as was done in Ref. [23]) but use classical rules applied to individual realizations of the microscopic quantum model.

We first discuss the construction of the classical problem. The Hamiltonian of a quantum many-body system can be expressed as a tight-binding model in Fock space [24], with the form

$$
\mathcal{H}=\sum_{I} \mathcal{E}_{I}|I\rangle\left\langle I\left|+\sum_{I \neq K} T_{I K}\right| I\right\rangle\langle K|,
$$

where $\{|I\rangle\}$ denotes a set of many-body basis states. We consider a graph in Fock space, consisting of nodes that represent these basis states and edges that indicate nonzero matrix elements $T_{I K}$. A suitable choice of basis is the one corresponding asymptotically to eigenstates in the strong disorder limit.

The percolation problem we study arises by designating edges active or inactive according to a microscopically based rule. An edge is defined to be active if and only if

$$
\left|T_{I K}\right|>\left|\mathcal{E}_{I}-\mathcal{E}_{K}\right| \text {. }
$$

This rule is motivated by the fact that for a two-state quantummechanical problem consisting of energy levels with separation $\Delta$ and coupling $J$, the extent of hybridization is controlled by the ratio $J / \Delta$. A percolation cluster is a maximal set of nodes joined by edges that are active, and a transition occurs in the model we treat because a decreasing proportion of edges are active as disorder strength is increased. 
An appropriate diagnostic for the phase transition in the classical model can be defined as follows, taking motivation from the behavior of participation entropies of quantum-mechanical eigenstates. For a many-body eigenstate $|\psi\rangle$ the first participation entropy in Fock space is $S_{1}=-\left.\sum_{I}|\langle\psi \mid I\rangle|^{2} \ln \langle\psi \mid I\rangle\right|^{2}$. Denoting the Fock-space dimension by $N_{\mathcal{H}}$, a characteristic feature of the delocalized phase is the scaling $S_{1} \sim a_{1} \ln N_{\mathcal{H}}$ with $a_{1}=1$. By contrast, in the localized phase, $a_{1}<1$ [11]. In a classical model, since there are no probabilities of the form $|\langle\psi \mid I\rangle|^{2}$, we define a scaled indicator function

$$
p_{I}= \begin{cases}1 / N_{\mathcal{C}}, & I \in \mathcal{C} \\ 0, & \text { otherwise }\end{cases}
$$

where $N_{\mathcal{C}}$ denotes the number of nodes in the cluster $\mathcal{C}$. The distribution of $p_{I}$ over Fock space plays an analogous role to probabilities derived from a quantum wave function, and the equivalent of the participation entropy is

$$
\overline{-\sum_{I} p_{I} \ln p_{I}}=\overline{\ln N_{\mathcal{C}}}=\ln \mathcal{S}_{\mathrm{typ}} .
$$

This is simply the logarithm of the typical cluster size $\mathcal{S}_{\text {typ }}$, with an average over disorder realizations denoted by $\overline{(\cdot)}$. For completeness we also define the average cluster size $\mathcal{S}_{\text {avg }}=\overline{N_{\mathcal{C}}}$. In analogy with participation entropies, we expect $\mathcal{S}_{\text {avg/typ }} \sim N_{\mathcal{H}}^{\alpha_{\text {avg/typ }}}$ with $\alpha_{\text {avg/typ }}=1$ in the percolating (delocalized) phase and $\alpha_{\text {avg/typ }}<1$ in the nonpercolating (localized) phase. Hence we use the value of $\alpha_{\text {avg/typ }}$ as a diagnostic for the phase transition.

To put the entire formulation on a concrete footing we consider a quantum Ising chain of spins $1 / 2$ with disordered longitudinal and uniform transverse fields, described by the Hamiltonian

$$
\mathcal{H}=J_{z} \sum_{\ell=1}^{N-1} \sigma_{\ell}^{z} \sigma_{\ell+1}^{z}+\sum_{\ell=1}^{N} h_{\ell} \sigma_{\ell}^{z}+J \sum_{\ell=1}^{N} \sigma_{\ell}^{x},
$$

where $\sigma_{l}^{z}= \pm 1$, and $h_{\ell} \in[-W, W]$ are random fields drawn from a uniform distribution. As the disorder couples to the $\left\{\sigma_{\ell}^{z}\right\}$, a natural choice for $\{|I\rangle\}$ is the basis composed of product states with $\sigma_{\ell}^{z}= \pm 1$. Since the off-diagonal part of $\mathcal{H}$ is simply $\sum_{\ell} \sigma_{\ell}^{x}$, the Fock-space graph is precisely an $N$-dimensional hypercube with edges between pairs of spin configurations that differ only on one spin, as illustrated in Fig. 1. The Fock-space node energies $\mathcal{E}_{I}=\left\langle I\left|J_{z} \sum_{\ell} \sigma_{\ell}^{z} \sigma_{\ell+1}^{z}+h_{\ell} \sigma_{\ell}^{z}\right| I\right\rangle$ can be evaluated straightforwardly with our basis choice, and the energy difference $\left|\mathcal{E}_{I}-\mathcal{E}_{K}\right|$ between states connected by spin reversal at site $\ell$ is $2\left|J_{z}\left(\sigma_{\ell+1}^{z}+\sigma_{\ell-1}^{z}\right)+h_{\ell}\right|$. This includes both an on-site term and cooperative contributions that depend on the states of neighboring spins. To ensure that all edges are active in the weak disorder limit $(W \rightarrow 0)$ we require $J>4 J_{z}$. The corresponding quantum model has a many-body localization transition as a function of $W$ (see Supplemental Material [25] for details).

Before presenting our analytic treatment of this model, we show numerical results for $\mathcal{S}_{\text {avg/typ }}$ obtained by exact enumeration of clusters in a finite system [26]. From Fig. 2, it is clear that there is a critical disorder strength $W_{c}$. For $W<$ $W_{c}$ we find $\mathcal{S}_{\text {avg/typ }} \sim N_{\mathcal{H}}$, indicating a percolating phase. For
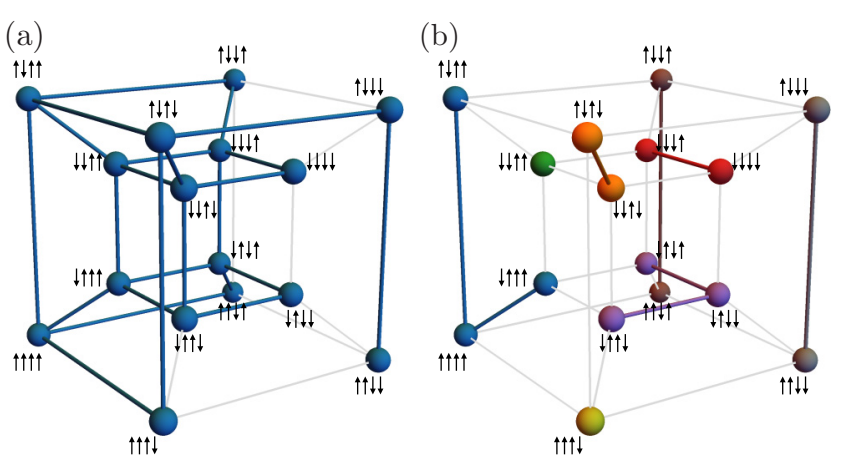

FIG. 1. Caricatures of (a) the percolating (delocalized) and (b) the nonpercolating (localized) phases for a system of four spins $1 / 2$. The nodes represent the $2^{4}=16$ basis states of the Fock space as indicated by the spin configurations. All nodes in a given cluster, and the active edges joining them, have the same color. Edges colored gray are inactive: they represent nonzero matrix elements in the quantum Hamiltonian that fail to satisfy the percolation criterion of Eq. (2).

$W>W_{c}$ we find a localized phase in which $\mathcal{S}_{\text {avg/typ }} \sim N_{\mathcal{H}}^{\alpha_{\text {avg/yp }}}$ with $\alpha<1$. The latter scaling is akin to the behavior of quantum eigenstates in the many-body localized phase $[11,16]$. The values of $W_{c}$ and $v$ can be obtained by collapsing the data for various system sizes onto a universal function $g\left[\left(W-W_{c}\right) N^{1 / \nu}\right]$. The data for $\mathcal{S}_{\text {avg }}$ yields $W_{c} \approx 1.95$ and $v \approx$ 1.79 , whereas those for $\mathcal{S}_{\text {typ }}$ give $W_{c} \approx 1.94$ and $v \approx 1.81$. These results are close to the exact values $W_{c}=J / 2=2.05$ and $v=2$ derived below.

An important further numerical observation is that in the percolating phase all Fock-space nodes form a single cluster, as indicated by the fact that $\mathcal{S}_{\text {avg/typ }}$ not only scales linearly with $N_{\mathcal{H}}$ but is equal to it. This behavior is completely different from that for bond percolation on a finite-dimensional lattice. It arises because of the high coordination number $(=N)$ of the Fock-space graph, and despite the fact that for $W \lesssim W_{c}$ a finite fraction of edges are inactive. Both the formation of a single cluster and the presence of inactive edges in the percolating phase are central to our analytical approach, which we sketch below with further details in [25].
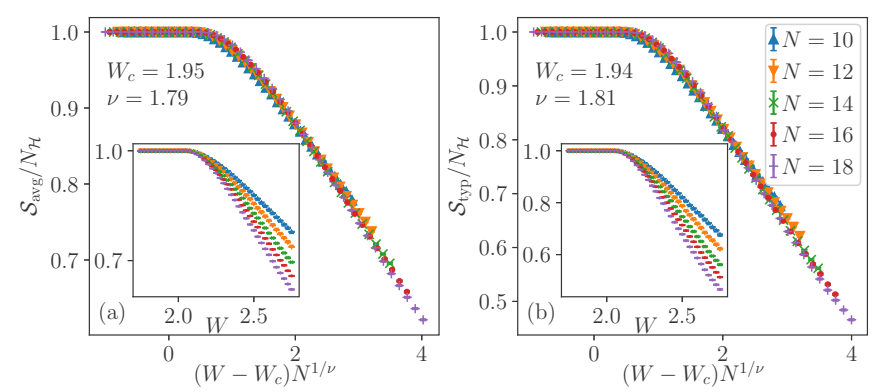

FIG. 2. Average (a) and typical (b) cluster sizes, normalized by Fock-space dimension, as a function of disorder strength for various system sizes $N$. Insets show raw data and main panels show scalecollapsed data with best fits for $W_{c}$ and $\nu$. Calculations use $J_{z}=1$ and $J=4.1$ with $10^{5}$ realizations and errors estimated via the standard bootstrap method with 500 resamplings. 


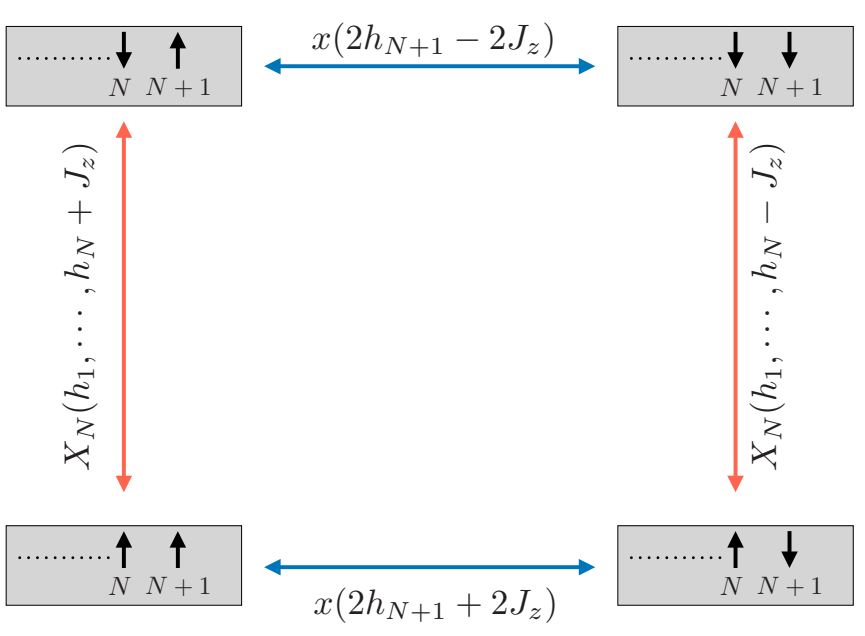

FIG. 3. Schematic representation of transitions involving spin flips at sites $N$ and $N+1$.

To indicate the existence of a single cluster, we consider in a system of size $N$ a function of the disorder realization with the properties

$$
X_{N}\left(\left\{h_{\ell}\right\}\right)= \begin{cases}1, & \text { if } N_{\mathcal{C}}=N_{\mathcal{H}} \\ 0, & \text { otherwise }\end{cases}
$$

Our strategy is to relate systems with open boundary conditions of size $N$ and $N+1$, by writing an expression for $X_{N+1}$ in terms of $X_{N}$ and $h_{N+1}$. This constitutes our basic recursion relation. We then perform a disorder average over all the fields and solve the averaged recursion relation to obtain the probability that all the nodes of the Fock-space graph belong to the same cluster. In this way we identify the critical point and determine the localization length exponent.

To find a recursion relation, we must account for two points. First, the edge representing a spin flip at site $N+1$ may be active or inactive, depending both on the value of $h_{N+1}$ and (via the exchange interaction) on the spin orientation at site $N$. Second, the effective field $h_{N}$ acting on the spin at site $N$ is modified in the $N+1$ site system by the exchange interaction with the spin at site $N+1$. To represent these features we define the indicator function

$$
x(\Delta)= \begin{cases}1, & |\Delta|<J \\ 0, & \text { otherwise }\end{cases}
$$

where $\Delta=\mathcal{E}_{I}-\mathcal{E}_{K}$ for configurations $|I\rangle$ and $|K\rangle$ connected by a given spin flip. Transitions on the Fock-space graph arising from spin flips at sites $N$ and $N+1$ are shown schematically in Fig. 3. Those denoted with blue arrows arise from spin flips at site $N+1$ and are influenced by the exchange field only from the spin at site $N$. Hence $\Delta=2 h_{N+1}+2 J_{z} \sigma_{N}^{z}$ in this case. Transitions denoted with red arrows arise from spin flips at site $N$ and have $\Delta=2\left(h_{N}+J_{z} \sigma_{N+1}^{z}\right)+2 J_{z} \sigma_{N-1}^{z}$. Here the exchange interaction with the spin at site $N+1$ is equivalent to the modification $h_{N} \rightarrow h_{N}+J_{z} \sigma_{N+1}^{z}$ of the field on site $N$.

In order that the four nodes represented in Fig. 3 are connected, it is sufficient that at least three of the edges depicted are active. From this we derive the recursion relation

$$
\begin{aligned}
X_{N+1}= & X_{N}^{+} X_{N}^{-}\left(x_{N+1}^{+}+x_{N+1}^{-}-3 x_{N+1}^{+} x_{N+1}^{-}\right) \\
& +\left(X_{N}^{+}+X_{N}^{-}\right) x_{N+1}^{+} x_{N+1}^{-} .
\end{aligned}
$$

Here $X_{N}^{ \pm}=X_{N}\left(h_{1}, \ldots, h_{N} \pm J_{z}\right)$ and $x_{N}^{ \pm}=x\left(2 h_{N} \pm 2 J_{z}\right)$. To obtain a closed set of equations we require expressions for $X_{N+1}^{ \pm}$. These are simple to obtain because in Eq. (8) the field $h_{N+1}$ enters the expression for $X_{N+1}$ only via $x_{N+1}^{ \pm}$, which is independent of the fields at other sites. Hence equations for $X_{N+1}^{ \pm}$are obtained by replacing $h_{N+1}$ with $h_{N+1} \pm J_{z}$ in the argument of $x_{N+1}^{ \pm}$. Defining the notation $z_{N}^{ \pm}=x\left(2 h_{N} \pm 4 J_{z}\right)$, $z_{N}=x\left(2 h_{N}\right)$, and $Y_{N}\left(\left\{h_{\ell}\right\}\right)=X_{N}^{+} X_{N}^{-}$, the recursion relations for $X_{N+1}^{ \pm}$are

$$
\begin{aligned}
X_{N+1}^{ \pm}= & Y_{N}\left(z_{N+1}+z_{N+1}^{ \pm}-3 z_{N+1} z_{N+1}^{ \pm}\right) \\
& +\left(X_{N}^{+}+X_{N}^{-}\right) z_{N+1} z_{N+1}^{ \pm} .
\end{aligned}
$$

Finally, we can find an expression for $Y_{N+1}$ from Eq. (9) by multiplying the recursions relations for $X_{N+1}^{+}$and $X_{N+1}^{-}$, giving

$$
\begin{aligned}
Y_{N+1}= & Y_{N}\left[z_{N+1}+z_{N+1}^{+} z_{N+1}^{-}-3 z_{N+1} z_{N+1}^{+} z_{N+1}^{-}\right] \\
& +\left(X_{N}^{+}+X_{N}^{-}\right) z_{N+1} z_{N+1}^{+} z_{N+1}^{-} .
\end{aligned}
$$

Linearity in $Y_{N+1}$ and $X_{N}^{ \pm}$is retained under multiplication because $Y_{N}$ and $X_{N}^{ \pm}$are idempotent.

The recursion relations (9) and (10) are a closed set of coupled linear equations for $Y_{N}$ and $X_{N}^{ \pm}$. They have the form $\mathbf{v}_{N+1}=\mathbf{M}_{N+1} \mathbf{v}_{N}$ where $\mathbf{v}_{N}=\left(Y_{N}, X_{N}^{+}, X_{N}^{-}\right)^{T}$. Crucially, the matrix $\mathbf{M}_{N+1}$ depends only on $h_{N+1}$ and not on $h_{\ell}$ for $\ell \leqslant N$. Due to this and the linearity, the system of equations can be converted into one for the averaged quantities. Defining the disorder average by $\overline{(\cdot)}=\left[\prod_{\ell=1} \int d h_{\ell} P\left(h_{\ell}\right)\right](\cdot)$ where $P(h)=\Theta(W-|h|) / 2 W$, the averaged system of equations is

$$
\overline{\mathbf{v}}_{N+1}=\overline{\mathbf{M}} \cdot \overline{\mathbf{v}}_{N}=(\overline{\mathbf{M}})^{N} \cdot \overline{\mathbf{v}}_{1},
$$

with $\overline{\mathbf{v}}_{1}=(1,1,1)^{T}$ as the boundary condition. From Eq. (11), $\overline{\mathbf{v}}_{N}$ can be obtained for arbitrary $N$. Substitution into the disorder-averaged form of Eq. (8) gives $\bar{X}_{N}$. This locates the phase transition: $\bar{X}_{N}$ is unity for all $N$ in the percolating phase, and decreases with increasing $N$ in the localized phase.

The critical disorder strength and localization length exponent can be evaluated analytically by computing the eigenvalues of $\overline{\mathbf{M}}$. We expect the largest eigenvalue, denoted by $\lambda_{\max }$, to be unity in the percolating phase and smaller than unity in the localized phase. We indeed find [25]

$$
\lambda_{\max }= \begin{cases}1 & \text { for } W \leqslant J / 2 \\ \Lambda<1 & \text { for } J / 2<W\end{cases}
$$

with $\Lambda=\left\{J+4 J_{z}+\left[\left(J-4 J_{z}\right)\left(8 W-3 J-4 J_{z}\right)\right]^{1 / 2}\right\} / 4 W$ for $J / 2 \leqslant W<\left(J+4 J_{z}\right) / 2$. This shows that the critical disorder strength is $W_{c}=J / 2$. The value of the exponent $v$ is determined by the asymptotic form

$$
1-\lambda_{\max }(W) \sim\left(W-W_{c}\right)^{v} \quad \text { for } W \gtrsim W_{c} .
$$

Expanding Eq. (12) around $W_{c}=J / 2$ for $W>J / 2$, we find

$$
\lambda_{\max }=1-\frac{4}{J\left(J-4 J_{z}\right)}\left(W-W_{c}\right)^{2}+O\left[\left(W-W_{c}\right)^{3}\right] .
$$


From this we identify the value $v=2$. The analytical determination of $W_{c}$ and $v$ constitutes two of our central results.

Understanding of the critical point is provided by a simple picture for the appearance of short, real-space segments in the chain where spins cannot fluctuate. As an example, consider three consecutive sites, $\ell-1, \ell$, and $\ell+1$. Suppose that $\left|2 h_{\ell \pm 1}\right|>J$ and $\left|2 h_{\ell}+4 J_{z}\right|>J$. Then the spins at these sites in a Fock-space cluster with $\sigma_{\ell-1}^{z}=\sigma_{\ell}^{z}=\sigma_{\ell+1}^{z}=1$ are frozen: the cluster has no active edges to nodes with other configurations of these spins, regardless of the orientation of the spins at sites $\ell \pm 2$. Such a disorder realization requires fields on two end sites ( $h_{\ell \pm 1}$ in this case) exceeding a critical strength $W_{c}=J / 2$. Similar arguments also apply if the end sites are adjacent, or if they have more than one site separating them. Frozen segments therefore appear with a density proportional to $\left(W-W_{c}\right)^{2}$ and their separation defines a correlation length, implying $v=2$.

We note that the value $v=2$ is consistent with the HarrisCCFS bound [27,28], which stipulates for a disorder-driven phase transition that $v \geqslant 2 / d$ in spatial dimension $d$. A similar bound has been derived for many-body localization transitions $[29,30]$. It is interesting that a scaling theory of entanglement at the many-body localization transition, based on treating the many-body resonances classically in real space, also yields $v=2$ for the typical data [19]. Note that exact diagonalization studies for this model in practice yield $v \approx 1$ [25] (as they do for various other models in 1D), which is in violation of the Harris-CCFS bounds.

It is natural to ask how important a role the exchange interaction $J_{z}$ plays in the transition, particularly since $W_{c}$ is independent of $J_{z}$. In fact, the character of the localized phase is controlled by interactions. This is illustrated most directly by the fact that $v$ is discontinuous, taking the value $v=1$ at $J_{z}=0$ [25]. It is revealed in more detail by considering the magnetization $m$ at a given real-space site, averaged over all nodes in a Fock-space cluster, and the probability distribution $P_{m}(m)$ of this quantity over disorder realizations. In the percolating phase $P_{m}(m)=\delta(m)$, while in the localized phase without interactions $P_{m}(m)$ has delta-function components at $m=0$ and $m= \pm 1$. As illustrated in Fig. 4, interactions
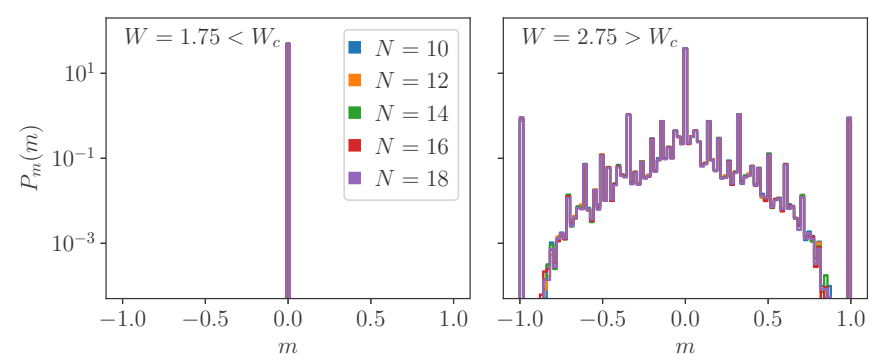

FIG. 4. Probability distribution of the cluster-averaged magnetization in the percolating (left) and localized (right) phases, computed by complete enumeration of clusters.

generate a broad background in addition to the delta-function components, which we study in detail elsewhere [31]. Analogous differences in the distributions of eigenstate expectation values of local observables have also been observed across the many-body localization transition [32,33].

In summary, we have constructed a classical percolation model in the Fock space of a disordered, interacting quantum spin chain. The classical model mimics aspects of the manybody localization transition in the quantum system. Using a transfer matrix approach we have computed exactly for the classical problem the critical disorder strength and localization length exponent. We have corroborated these results by enumerating clusters exactly on finite-sized systems.

A number of interesting directions remain open. In particular, the Fock-space percolation model can be interpreted as an example of a kinetically constrained model. Such models are known to show dynamical phase transitions and nonergodic behavior [34-37]. They can be studied using Monte Carlo dynamics, which gives access to much larger system sizes than complete enumeration. This perspective also leads to the introduction of new, dynamical correlation functions, which we examine in a separate work [31].

We would like to thank S. Gopalakrishnan, A. Nahum, and S. A. Parameswaran for useful discussions. This work was in part supported by EPSRC Grant No. EP/N01930X/1.
[1] M. Suzuki, Relationship between $d$-dimensional quantal spin systems and $(d+1)$-dimensional Ising systems: Equivalence, critical exponents and systematic approximants of the partition function and spin correlations, Prog. Theor. Phys. 56, 1454 (1976)

[2] S. Sachdev, Quantum Phase Transitions (Cambridge University Press, Cambridge, UK, 2011).

[3] M. Tsukada, On the tail states of the Landau subbands in MOS structures under strong magnetic field, J. Phys. Soc. Jpn. 41, 1466 (1976).

[4] R. F. Kazarinov and S. Luryi, Quantum percolation and quantization of Hall resistance in two-dimensional electron gas, Phys. Rev. B 25, 7626 (1982).

[5] S. A. Trugman, Localization, percolation, and the quantum Hall effect, Phys. Rev. B 27, 7539 (1983).

[6] D. M. Basko, I. L. Aleiner, and B. L. Altshuler, Metal-insulator transition in a weakly interacting many-electron system with localized single-particle states, Ann. Phys. (NY) 321, 1126 (2006).

[7] I. V. Gornyi, A. D. Mirlin, and D. G. Polyakov, Interacting Electrons in Disordered Wires: Anderson Localization and Low-T Transport, Phys. Rev. Lett. 95, 206603 (2005).

[8] V. Oganesyan and D. A. Huse, Localization of interacting fermions at high temperature, Phys. Rev. B 75, 155111 (2007).

[9] M. Žnidarič, T. Prosen, and P. Prelovšek, Many-body localization in the Heisenberg $X X Z$ magnet in a random field, Phys. Rev. B 77, 064426 (2008).

[10] A. Pal and D. A. Huse, Many-body localization phase transition, Phys. Rev. B 82, 174411 (2010).

[11] D. J. Luitz, N. Laflorencie, and F. Alet, Many-body localization edge in the random-field Heisenberg chain, Phys. Rev. B 91, 081103(R) (2015). 
[12] J. A. Kjäll, J. H. Bardarson, and F. Pollmann, Many-Body Localization in a Disordered Quantum Ising Chain, Phys. Rev. Lett. 113, 107204 (2014).

[13] R. Nandkishore and D. A. Huse, Many-body localization and thermalization in quantum statistical mechanics, Annu. Rev. Condens. Matter Phys. 6, 15 (2015).

[14] J. Z. Imbrie, On many-body localization for quantum spin chains, J. Stat. Phys. 163, 998 (2016).

[15] D. Stauffer and A. Aharony, Introduction to Percolation Theory, revised 2nd ed. (CRC Press, Boca Raton, FL, 1994).

[16] A. De Luca and A. Scardicchio, Ergodicity breaking in a model showing many-body localization, Europhys. Lett. 101, 37003 (2013).

[17] R. Vosk, D. A. Huse, and E. Altman, Theory of the ManyBody Localization Transition in One-Dimensional Systems, Phys. Rev. X 5, 031032 (2015).

[18] A. C. Potter, R. Vasseur, and S. A. Parameswaran, Universal Properties of Many-Body Delocalization Transitions, Phys. Rev. X 5, 031033 (2015).

[19] P. T. Dumitrescu, R. Vasseur, and A. C. Potter, Scaling Theory of Entanglement at the Many-Body Localization Transition, Phys. Rev. Lett. 119, 110604 (2017).

[20] A. Goremykina, R. Vasseur, and M. Serbyn, Analytically Solvable Renormalization Group for the Many-Body Localization Transition, Phys. Rev. Lett. 122, 040601 (2019).

[21] P. T. Dumitrescu, A. Goremykina, S. A. Parameswaran, M. Serbyn, and R. Vasseur, Kosterlitz-Thouless scaling at many-body localization phase transitions, Phys. Rev. B 99, 094205 (2019).

[22] A. Chandran and C. R. Laumann, Semiclassical limit for the many-body localization transition, Phys. Rev. B 92, 024301 (2015).

[23] D. E. Logan and S. Welsh, Many-body localization in Fock space: A local perspective, Phys. Rev. B 99, 045131 (2019).

[24] S. Welsh and D. E. Logan, Simple probability distributions on a Fock-space lattice, J. Phys.: Condens. Matter 30, 405601 (2018).

[25] See Supplemental Material at http://link.aps.org/supplemental/ 10.1103/PhysRevB.99.220201 for the detailed derivation.
[26] For definiteness, we consider the cluster that contains the node $I_{0}$ with $\mathcal{E}$ closest to the mean, so that $\mathcal{E}_{I_{0}}=\min _{I}\left\{\left|\mathcal{E}_{I}-\overline{\mathcal{E}}\right|\right\}$ where $\overline{\mathcal{E}}=\sum_{I} \mathcal{E}_{I} / N_{\mathcal{H}}$.

[27] A. B. Harris, Effect of random defects on the critical behavior of Ising models, J. Phys. C: Solid State Phys. 7, 1671 (1974).

[28] J. T. Chayes, L. Chayes, D. S. Fisher, and T. Spencer, FiniteSize Scaling and Correlation Lengths for Disordered Systems, Phys. Rev. Lett. 57, 2999 (1986).

[29] A. Chandran, C. R. Laumann, and V. Oganesyan, Finite size scaling bounds on many-body localized phase transitions, arXiv:1509.04285.

[30] V. Khemani, S. P. Lim, D. N. Sheng, and D. A. Huse, Critical Properties of the Many-Body Localization Transition, Phys. Rev. X 7, 021013 (2017).

[31] S. Roy, J. T. Chalker, and D. E. Logan, Percolation in Fock space as a proxy for many-body localization, Phys. Rev. B 99, 104206 (2019).

[32] C. L. Baldwin, C. R. Laumann, A. Pal, and A. Scardicchio, The many-body localized phase of the quantum random energy model, Phys. Rev. B 93, 024202 (2016).

[33] D. J. Luitz, Long tail distributions near the many-body localization transition, Phys. Rev. B 93, 134201 (2016).

[34] D. Sherrington, L. Davison, A. Buhot, and J. P. Garrahan, Glassy behavior in simple kinetically constrained models: Topological networks, lattice analogues and annihilation-diffusion, J. Phys.: Condens. Matter 14, 1673 (2002).

[35] J. P. Garrahan, R. L. Jack, V. Lecomte, E. Pitard, K. van Duijvendijk, and F. van Wijland, Dynamical First-Order Phase Transition in Kinetically Constrained Models of Glasses, Phys. Rev. Lett. 98, 195702 (2007).

[36] F. Ritort and P. Sollich, Glassy dynamics of kinetically constrained models, Adv. Phys. 52, 219 (2003).

[37] J. P. Garrahan, P. Sollich, and C. Toninelli, Kinetically constrained models, in Dynamical Heterogeneities in Glasses, Colloids, and Granular Media, edited by L. Berthier, G. Biroli, J.-P. Bouchaud, L. Cipelletti, and W. van Saarloos (Oxford University Press, Oxford, 2011). 